\title{
Influence of External Acoustic Noise on the Operation of an Open Photoacoustic Helmholtz Cell
}

\begin{abstract}
T. STARECKI
Institute of Electronic Systems, Warsaw University of Technology

Nowowiejska 15/19, 00-665 Warsaw, Poland

One of the most important problems in the design of open photoacoustic cells is appropriate attenuation of the external acoustic noise, because in the case of weak photoacoustic signals even relatively low level of such an acoustic noise can severely limit sensitivity of the setup. It is important that the mentioned filtering is not limited to the frequencies close to the light modulation frequency, but performed in the possibly widest band width, because although it is usually considered that setup sensitivity depends directly on the parameters of the final narrowband filtering and/or synchronous detection, it should be noticed that it can be also seriously affected by saturation of the signal amplifiers being overdriven by the signal components resulting from the mentioned acoustic noise. The paper describes how influence of the external acoustic noise on the operation of an open photoacoustic Helmholtz cell depends on mechanical dimensions of the cell. The results show that appropriate selection of the dimensions results in noticeable improvement of the external acoustic noise attenuation.
\end{abstract}

PACS numbers: 43.50.Gf, 43.38.Zp, 43.58.Kr

\section{Introduction}

One of the most important components of nearly all photoacoustic setups is a photoacoustic cell. In the simplest case it is an enclosed container in which the investigated sample is placed. However, the main disadvantage of such a solution is necessity of partial disassembling of the cell (e.g. unscrewing a window) every time when the sample must be replaced. If the cell is applied in fluid measurements, it can be equipped with valves and pumps run periodically when replacement of the sample is required, or it can be designed as an open cell, which has direct connection with the exterior. The latter solution usually makes use of the cell and its design much simpler (valves can be removed, the fluid flow can be forced or spontaneous, etc.), but has a strong disadvantage of strongly increased feedthrough of the external acoustic noise to the interior of the cell, which may substantially reduce sensitivity of the setup. 


\section{Attenuation of the external acoustic noise in the open cell designs}

Open photoacoustic resonators are usually based on standing wave resonances [1-4], while typical approaches to the attenuation of the acoustic noise use acoustic filters, baffles and quarterwave separating ducts (see e.g. Figs. 1 and 2). The cell can be also implemented as a Helmholtz resonator, e.g. as in Fig. 3. Such a design simplifies implementation of continuous flow cells, and, if the dimensions of the inlet/outlet ducts are selected properly, Q-factor of such a cell is comparable with a Q-factor of corresponding close Helmholtz structure. It may seem at first
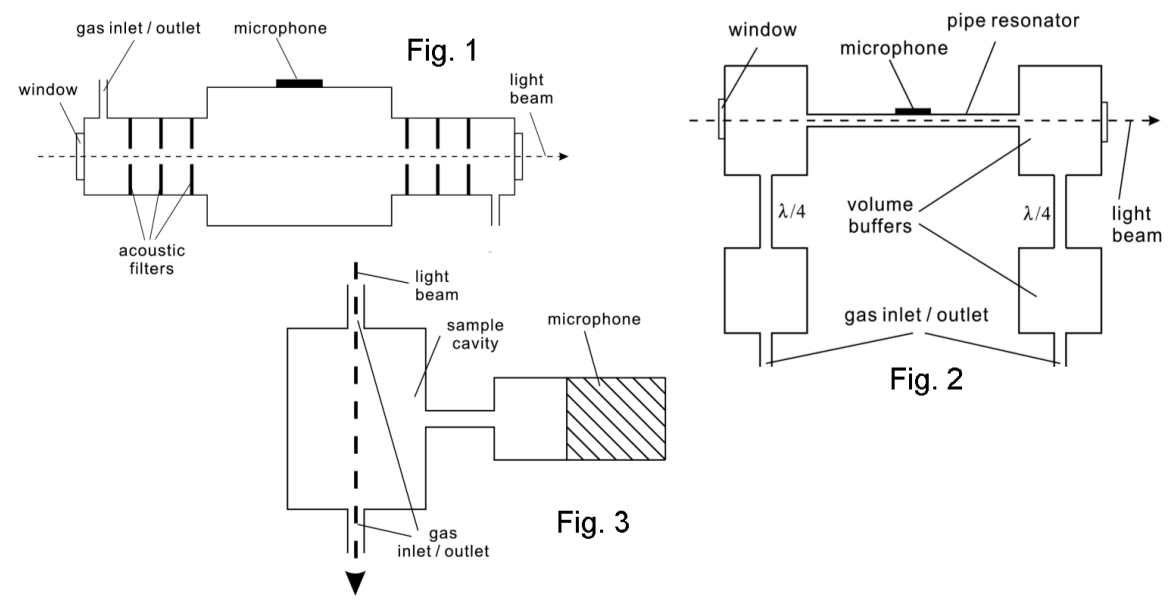

Fig. 2

Fig. 1. Example of a high-Q resonant cell with acoustic filters [5].

Fig. 2. A longitudinal resonator with volume buffers and quarterwave acoustic filters $[6]$.

Fig. 3. A windowless open Helmholtz cell [7].

that in the case of a Helmholtz structure it is also enough to properly select length of the duct connecting the cell with the exterior, in order to obtain similar attenuation of the external acoustic noise as in the case of the quarterwave filters from Fig. 2. However, it must be taken into account that quarterwave transmission line behaves like a very high acoustic impedance only if the other end of the line is connected to a low acoustic impedance structure - e.g. a high volume cavity (as in the case of the cell from Fig. 2), as

$$
Z_{\mathrm{i}}=\frac{Z_{w}^{2}}{Z_{l}}
$$

where $Z_{\mathrm{i}}, Z_{\mathrm{l}}$ and $Z_{w}$ are respectively: input, load, and characteristic impedance of the line. As a result, a quarterwave duct in the Helmholtz resonator from Fig. 3 will represent high acoustic impedance only from the sample cavity side, while, due to relatively small size of this cavity, from the exterior the duct will behave like an impedance of another (much lower value). This means that leakage of the 
photoacoustic signal from the sample cavity to the exterior will be small, but external acoustic noise will strongly propagate to the interior of the cell. In such a case it is of primary importance to know if it is possible to minimize the mentioned effect (external noise penetration) by appropriate selection of the duct's length and diameter, and without negative impact on the frequency response of the cell (in particular on its Q-factor).

The most convenient method that can be used to evaluate attenuation of the external acoustic noise in photoacoustic open Helmholtz resonators is computer simulation, which allows for quick change of cell dimensions and investigation of their influence on the frequency response. For this purpose a loss-improved model [8] (that had been previously verified on multi-cavity structures of similar dimensions) was used. Results of the simulations for the cells with sample and microphone cavities of respectively $2 \mathrm{~cm}^{3}$ and $1.5 \mathrm{~cm}^{3}$ connected with a duct $3 \mathrm{~cm}$ in length and $3 \mathrm{~mm}$ in diameter are shown in Fig. 4. The figure presents charac-

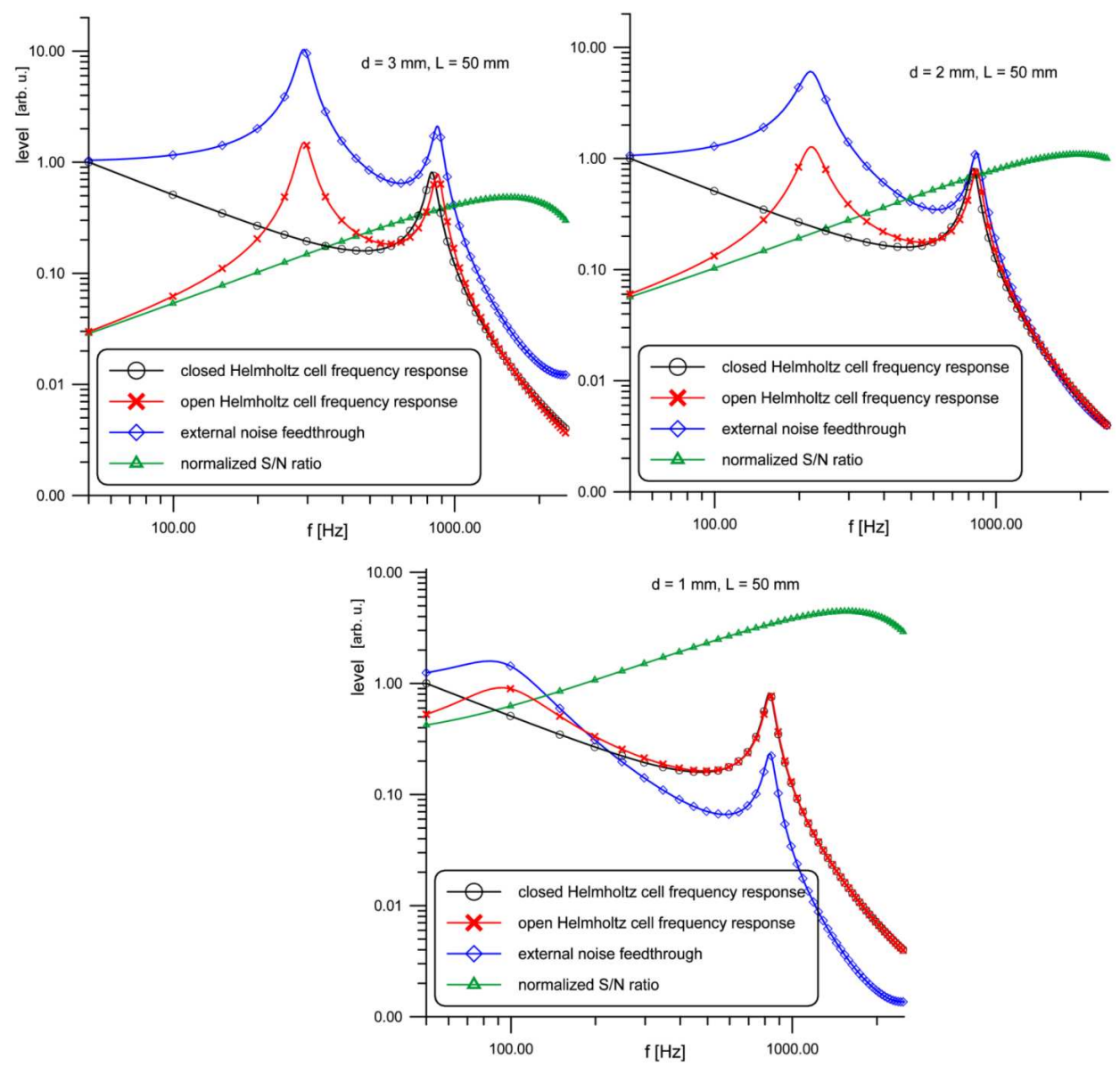

Fig. 4. Influence of diameter of inlet/outlet ducts on characteristics of the open Helmholtz cell. 
teristics for different values of diameter of the inlet/outlet ducts and contains:

- frequency responses of the cells,

- frequency response of the corresponding closed cell (given as a reference),

- level of the external acoustic noise feedthrough, given as level of the noise measured in the microphone cavity, and under assumption that the external noise is white,

- signal-to-noise ratio calculated as a ratio of the already mentioned frequency response of the cell and external noise feedthrough characteristics.

Presented results show that in the case of open photoacoustic Helmholtz resonators there is a strong feedthrough of the external acoustic noise to the microphone cavity, and that until the second resonance is reached (and even a bit further) the signal-to-noise ratio improves with the frequency. It can be also easily noticed that in terms of attenuation of the external acoustic noise and maximum signal gain, the optimal frequency of operation is the second resonance frequency, as further increase in the frequency results in strong decrease in the output signal with only slight improvement of the signal-to-noise ratio.

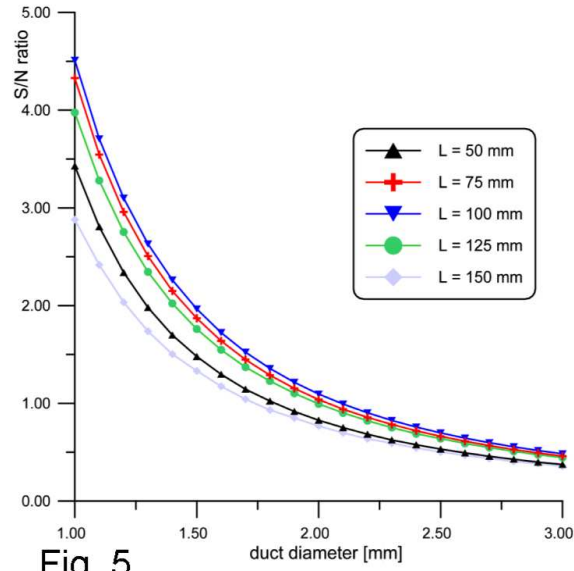

Fig. 5

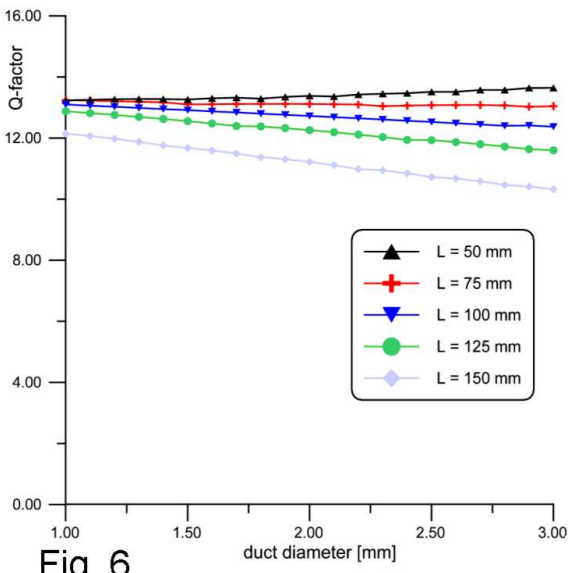

Fig. 6

Fig. 5. Signal-to-noise ratio vs. inlet/outlet duct diameter.

Fig. 6. Q-factor vs. inlet/outlet duct diameter.

In order to evaluate optimal length and diameter of the opening ducts for the given Helmholtz structure, influence of these parameters on the signal-to-noise ratio and $\mathrm{Q}$-factor of the cell working at the frequency of the second resonance were calculated (Figs. 5-8). It is clearly visible from the figures that attenuation of the external acoustic noise improves with decrease in the inlet/outlet diameters. It can be also noticed that influence of the length and diameter of the inlet/outlet ducts on the Q-factor of the cell is small or negligible, and that signal-to-noise 


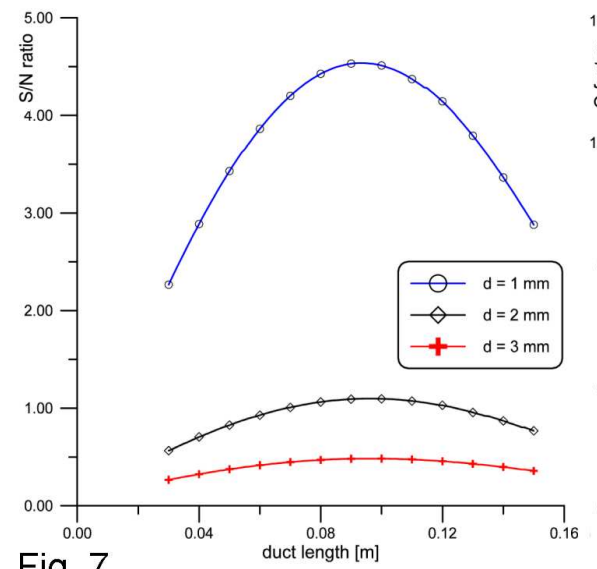

Fig. 7

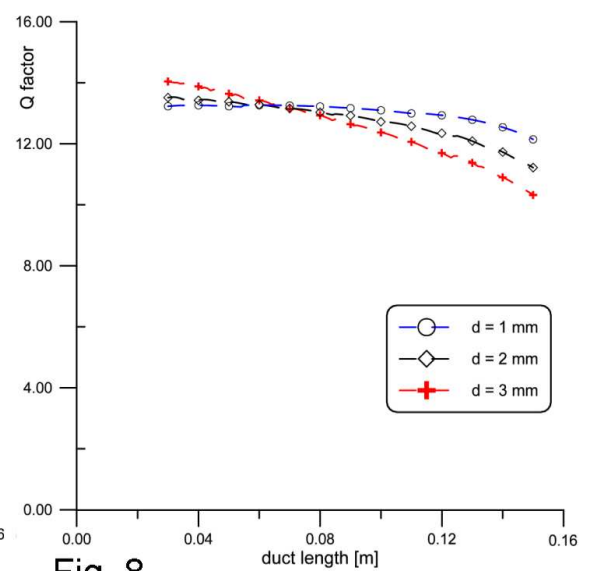

Fig. 8

Fig. 7. Signal-to-noise ratio vs. inlet/outlet duct length.

Fig. 8. Q-factor vs. inlet/outlet duct length.

ratio depends quite strong on length of the ducts, so that proper selection of the length can substantially improve attenuation of the external acoustic noise.

\section{Conclusions and remarks}

Presented analysis shows that in the case of an open photoacoustic Helmholtz cell external acoustic noise is an important factor that can severely limit sensitivity of the setup. Attenuation of the noise can be improved by appropriate selection of dimensions of the inlet/outlet ducts, in particular by making diameter of the ducts as small as possible. Practical limits of the diameter depend mainly on accuracy of collimation and axial positioning of the light beam. Optimal length of the inlet/outlet ducts depends on the frequency response of the internal cell structure, and it should be noticed that excessive length of the ducts increases complexity, cost and size of the setup without any improvement of the noise attenuation. For the investigated structure the optimal length was about $9 \mathrm{~cm}$ (approximately quarter of the acoustic wave that corresponded to the resonance frequency of the cell). However, even at the optimal length of the ducts and their diameter reduced to $1 \mathrm{~mm}$ external acoustic noise feedthrough was still significant, which means that such open Helmholtz cells can be applied in measurements of relatively strong photoacoustic signals with relatively low level of the external acoustic noise, or some additional means of protection against the noise must be applied.

\section{References}

[1] A. Miklós, A. Lörincz, Appl. Phys. B 48, 213 (1989).

[2] Z. Bozóki, J. Sneider, G. Szabó, A. Miklós, M. Serényi, G. Nagy, M. Fehér, Appl. Phys. B 63, 399 (1996). 
[3] A. Boschetti, D. Bassi, E. Iacob, S. Iannotta, L. Ricci, M. Scotoni, Appl. Phys. $B$ 74, 273 (2002).

[4] S. Schäfer, A. Miklós, P. Hess, Appl. Opt. 36, 3202 (1997).

[5] M.A. Gondal, A. Dastageer, M.H. Shwehdi, Talanta 62, 131 (2004).

[6] A. Schmohl, A. Miklós, P. Hess, Appl. Opt. 41, 1815 (2002).

[7] T. Starecki, Acta Phys. Pol. A 114, A-209 (2008).

[8] T. Starecki, J. Acoust. Soc. Am. 122, 2118 (2007). 